Revista Calidad en la Educación Superior

Programa de Autoevaluación Académica

Universidad Estatal a Distancia

ISSN 1659-4703

Costa Rica

revistacalidad@uned.ac.cr

\title{
ENFOQUES EN LOS MODELOS EDUCATIVOS, PLANES DE ESTUDIO Y SU CORRESPONDENCIA CON LA PLANEACIÓN DIDÁCTICA (DISEÑO INSTRUCCIONAL) EN LA EDUCACIÓN A DISTANCIA \\ FOCUS ON EDUCATIONAL MODELS, CURRICULUM AND TEACHING PLANNING CORRESPONDENCE (INSTRUCTIONAL DESIGN) IN DISTANCE EDUCATION
}

Rosita Ulate de Brooke ${ }^{1}$ Universidad Estatal a Distancia de Costa Rica

\author{
IV Edición \\ Volumen III, Número 2 \\ Noviembre 2011 \\ pp. $168-192$
}

Recibido: julio 2011

Aprobado: setiembre 2011

1 MBA., Asesora Académica Dirección de la Escuela de Ciencias Exactas y Naturales, correo electrónico: rulate@ uned.ac.cr 
Enfoques en los Modelos Educativos, Planes de Estudio y su Correspondencia con la Planeación Didáctica (Diseño Instruccional) en la Educación a Distancia,

Rosita Ulate

Inspirado en Bill y Esperanza Brooke.

\section{Resumen}

Es muy probable que los profesores universitarios, que no provienen del área de la educación, se sientan desprotegidos en su función docente al no contar con las bases pedagógicas necesarias para generar un proceso de enseñanza y aprendizaje exitoso, a pesar de estar a cargo de la formación de nuevos profesionales en su área de conocimiento. También es común que esos mismos profesores reciban en sus primeros años como docentes, una serie de capacitaciones en el campo de la pedagogía universitaria, diseños curriculares, producción de materiales, entre otros. Tanto la inducción como la capacitación es vital para comprender la gestión académica. Sin embargo he podido observar que muchas de las necesidades de los profesores se enfocan hacia soluciones prácticas en la planeación didáctica de las estrategias de aprendizaje, sean sincrónicas o asincrónicas.

Las estrategias de planeación didáctica desde los diferentes paradigmas de la educación y de los enfoques de las teorías de aprendizaje siguen siendo validas. Desde el conductismo, cognitivismo y constructivismo se han generado varios sistemas y modelos de diseño didáctico al que comúnmente se le ha conocido como sistemas de entrega del diseño instruccional. Algunos de los modelos son sumamente creativos, están disponibles, han sido utilizados y probados por la comunidad académica a través de las diversas innovaciones de los medios de comunicación e información (imprenta, radio, televisión, computadores, videos, internet, entre otros más de más recientes usos en la población mundial). Sin embargo modelos de sistemas de instrucción aún son desconocidos para muchos, especialmente los no formados en la educación, pero si responsables de la formación de generaciones de profesionales en su trabajo como docentes. 
Enfoques en los Modelos Educativos, Planes de Estudio y su Correspondencia con la Planeación Didáctica (Diseño Instruccional) en la Educación a Distancia,

Rosita Ulate

Mi experiencia empírica en la observación de actividades, talleres e investigaciones realizadas en el Programa de Protección y Manejo de Recursos Naturales (PROMARENA) de la Escuela de Ciencias Exactas y Naturales (ECEN) de la Universidad Estatal a Distancia (UNED), demuestra la necesidad de un sistema y de diseños instruccionales que concuerden con los enfoques y paradigmas dominantes de la carrera; así como con el diseño curricular del plan de estudios, de los cursos y de la evaluación de los aprendizajes.

Palabras clave: Teorías de aprendizaje, modelos de aprendizaje, diseño instruccional, estrategias de aprendizaje.

\section{Summary}

It is very likely that university professors who are not from the area of education, they feel vulnerable in their teaching by not having the necessary educational foundation to build a teaching and learning process successful, despite being in charge of training of new professionals in their area of expertise. It is also common that these same teachers receive in their first years as teachers, a series of trainings in the field of university pedagogy, curriculum design, and materials production, among others. Induction and training is vital to understand the academic administration. However I have noticed that many of the needs of teachers focus towards practical solutions in planning teaching-learning strategies, whether synchronous or asynchronous.

Educational planning strategies from different educational paradigms and approaches to learning theories are still valid. From behaviorism, cognitivist and constructivism have generated several systems and models of instructional design that has been commonly known as delivery systems of instructional design. Some of the models are highly creative, are available have been utilized and proven by the academic community through various innovations in media and information (print, 
Enfoques en los Modelos Educativos, Planes de Estudio y su Correspondencia con la Planeación Didáctica (Diseño Instruccional) en la Educación a Distancia,

Rosita Ulate

radio, television, computers, videos, internet, among others over uses the latest in the world population). But models of instruction sets are still unknown to many; especially those not trained in education, but if those responsible for training generations of professionals in their work as teachers.

My empirical experience in the observation of activities, workshops and research in the Agenda for Protection and Natural Resource Management (PROMARENA) School of Natural Sciences (ECEN) of the Universidad Estatal a Distancia (UNED), demonstrates the need and system designs that match instructional approaches and paradigms of the race, as well as curriculum design, courses and assessment of learning.

Keywords: Learning theories, learning models, instructional design, learning strategies.

\section{Introducción}

Durante mis años como docente en universidades presenciales no ameritaba mayor dificultad el estudiar un sílabo, seguir las indicaciones principales e impartir un curso de administración. Desde la libertad de la cátedra se da un permiso al docente para hacer ejercicio de su creatividad, emotividad y creencias con respecto a un tema determinado. El problema es que no todos los profesores gozan del arte del proceso de comunicación a pesar de ser diestros en la materia que enseñan. A los profesores sin ese don de enseñar, no les queda más que recurrir al método de prueba y error, sin cuantificar el costo de aquellos estudiantes que contribuyeron al error en el proceso de formación de un profesor sin el don de la enseñanza.

Gracias al sentido común de muchos profesionales, en diferentes áreas del conocimiento, que sin el mayor dominio de la pedagogía logran seguir un hilo 
Enfoques en los Modelos Educativos, Planes de Estudio y su Correspondencia con la Planeación Didáctica (Diseño Instruccional) en la Educación a Distancia,

Rosita Ulate

conductor con el que desempeñan un increíble trabajo en los procesos de enseñanza y aprendizaje. Sin embargo el don de ser maestro no es una máxima en todos los profesores universitarios. En el camino es necesario reconocer los errores y horrores a los que se ven sometidos los alumnos y los docentes.

Más difícil aún es cuando el modelo a seguir es de educación a distancia. En donde la interacción de la libre cátedra del profesor en el aula desaparece para convertirse en un equipo de trabajo que consolida, organiza y colabora en una serie de acciones, destinadas a que aquel alumno que de forma sincrónica o asincrónica logre su cometido y propósito de capacitación o desarrollo profesional de cara al compromiso con la sociedad y la comunidad mundial.

Parece que el auge del uso de modelos de educación ecléticos dejó de lado la importancia de enfocarse en los aspectos medulares de una buena planeación didáctica. Mi sorpresa es encontrar mallas de planes de estudio y diseños curriculares de cursos adecuadamente concebidos pero deficientes en la planeación didáctica, los cuales no garantizan la calidad y efectividad de la mediación pedagógica (instruccional). Efectividad, calidad y eficiencia en el proceso de enseñanza y aprendizaje que cada vez es imprescindible ante el auge de los sistemas de capacitación y formación profesional creados con uso de las plataformas de aprendizaje, sean de software libre o comercial. Especialmente cuando la rapidez y facilidad de herramientas tecnológicas permiten montar cursos en línea con una amplitud de recursos disponibles en internet que llevan a la saturación de información sin una mediación pedagógica.

¿Cómo se aseguran los profesionales en el campo de la educación, de la correspondencia entre el enfoque paradigmático dominante en un plan de estudios, el diseño curricular, el diseño instruccional y la evaluación de los aprendizajes? Al final la congruencia e interacción de estas partes del proceso de enseñanza y 
Enfoques en los Modelos Educativos, Planes de Estudio y su Correspondencia con la Planeación Didáctica (Diseño Instruccional) en la Educación a Distancia,

Rosita Ulate

aprendizaje son los responsables de las competencias, habilidades, destrezas, conocimientos y actitud que los estudiantes pondrán en práctica en el ejercicio de su profesión en la vida real.

\section{Los planes de estudio}

Aparentemente los diseños curriculares de los planes de estudio gozan de buenas iniciativas e intenciones, proceso que se plasma en la exposición del diseño curricular de un curso por medio de plantillas especialmente diseñadas para contemplar la información vital del curso. Hasta allí todo va bien, los objetivos, el temario está expuesto, nivel, nombre del cursos, estrategias de aprendizaje, evaluación. Lo preocupante es que en la gestión práctica del curso no todo es realizado como se planeó, es más me atrevería a asegurar que no hay una correspondencia observable desde el enfoque paradigmático del plan de estudios, el diseño curricular, los materiales de estudio, las estrategias y la evaluación de los aprendizajes.

Precisamente David Merrill (2008) contempla la importancia que al final de cada curso y de cada plan de estudios, los estudiantes puedan demostrar el logro de los objetivos en un contexto real. En algunas de las valoraciones hechas como asesora académica, me encuentro planes de estudio que asumen un enfoque ecléctico, promueven un diseño curricular por saberes o por perfil de salida, materiales didácticos con una mediación pedagógica conductista y evaluación de aprendizajes memorístico. Lamentablemente esa falta de correspondencia no permite una valoración del rendimiento académico y práctico del proceso de enseñanza y aprendizaje realizado. 
Enfoques en los Modelos Educativos, Planes de Estudio y su Correspondencia con la Planeación Didáctica (Diseño Instruccional) en la Educación a Distancia,

Rosita Ulate

\section{Creación de los programas de estudio y los enfoques de aprendizaje}

A comienzos del presente año 2011, se formó una comisióni en la ECEN con el propósito de dar seguimiento a los programas de estudio ofrecidos y su pertinencia con el modelo pedagógico de la UNED. Desde la óptica de una de las miembros se identifica el modelo pedagógico de la UNED como "un conjunto estructurado de principios y criterios que orientan la práctica educativa (...) de Carácter Ecléctico" (Azze, 2011, p.1). Paralelamente Azze (2011) presenta un cuadro descriptivo de los enfoques predominantes en los planes de estudio, con diferencias sustanciales disciplinarias pero con un acuerdo común entre todas a partir del modelo declaratorio de la UNED y con los componentes de educación centrada en el estudiante, una docencia desarrolladora, de aprendizaje auto-regulado, de evaluación, autoevaluación y comunicación didáctica (Véase Tabla 1).

Tabla 1: Análisis de particularidades del Modelo Pedagógico por Carrera de la ECEN. Adaptado de Azze, A. (2011)

\begin{tabular}{|c|c|c|}
\hline Carrera & Modelo Pedagógico & Particularidades del Currículo \\
\hline $\begin{array}{l}\text { Manejo de } \\
\text { Recursos } \\
\text { Naturales. }\end{array}$ & $\begin{array}{l}\text { Asume el Modelo en su concepción amplia y carácter y } \\
\text { los principios de la Educación de Adultos. }\end{array}$ & $\begin{array}{l}\text { Base Plan de Estudios: Formación humanistaii, } \\
\text { en ciencias, profesional y relación teórico } \\
\text { práctica. Introduce el Aprendizaje en línea }\end{array}$ \\
\hline $\begin{array}{l}\text { Programa } \\
\text { Enseñanza de } \\
\text { las Ciencias } \\
\text { Naturales }\end{array}$ & $\begin{array}{l}\text { Declara el énfasis en la concepción socio cultural del } \\
\text { aprendizaje (Vygotsky).Filosofía Educativa del } \\
\text { Construccionismo social y Teoría Constructivista } \\
\text { (Piaget). Define: Enfoque curricular } \\
\text { socioreconstructivismo (proceso de socialización y } \\
\text { culturalización). Potencia el Contexto Social como base a } \\
\text { la socialización y validación de la teoría con la realidad y } \\
\text { experiencia cotidiana, para explicar y solucionar } \\
\text { problemas. }\end{array}$ & $\begin{array}{l}\text { Base Plan de Estudios: Formación humanista, } \\
\text { en ciencias, profesión y relación teórico práctica. } \\
\text { Estrategia metodológica: Socio constructivista, } \\
\text { promueve la socialización y el trabajo en grupos, } \\
\text { la solución de problemas del contexto social e } \\
\text { introduce el aprendizaje en línea. }\end{array}$ \\
\hline
\end{tabular}


Enfoques en los Modelos Educativos, Planes de Estudio y su Correspondencia con la Planeación Didáctica (Diseño Instruccional) en la Educación a Distancia,

Rosita Ulate

\begin{tabular}{ll}
\hline Programa & Modelo Pedagógico de la UNED con énfasis en la \\
Enseñanza de la & Teoría Genética de J. Piaget, en los referentes a las \\
Matemática & estrategias, cognitivas y su epistemología constructivista \\
& y la concepción socio cultural del aprendizaje, tal como la \\
& plantean L.S Vygotsky, A. Luria, A. Leontiev y sus \\
& seguidores. La teoría del aprendizaje D. Ausubel. \\
& Teorías de aprendizaje como procesamiento de \\
& información. La Teoría de la Educación de Adultos.
\end{tabular}

Administración de Servicios de Salud.

Ing. Agronómica Asume un enfoque cognitivo-constructivista: $(E \mid$ constructivismo cognitivo se caracteriza por no contemplar el subjetivismo humano en el proceso de construcción de la realidad).

\section{Ingeniería \\ Modelo Pedagógico de la UNED.}

Agroindustrial

Ingeniería

Informática

Administración

Agropecuarias
Modelo Pedagógico de la UNED. Define el Modelo en su concepción amplia (Conjunto estructurado de principios y criterios que orientan la práctica educativa) y los principios de la Educación de Adultos.
Base Plan de Estudios: Formación humanista en ciencias, profesional y relación teórico práctica.

Introduce Aprendizaje en línea.
Base Plan de Estudios: Formación humanista, en ciencias, profesional y relación teórico práctica

Base Plan de Estudios: Formación humanista, en ciencias, profesional y relación teórico práctica

Base Plan de Estudios: Formación humanista en ciencias, profesional y relación teórico práctica.

Estrategia metodológica: La solución de problemas.

Plantea contextualizar y hacer funcional el aprendizaje. Fomentar la investigación y el uso de tecnologías limpias, que garanticen la conservación ambiental.

Base Plan de Estudios: Formación humanista, en ciencias, profesional, relación teórico práctica y relación profesión-desarrollo de la personalidad.

Estrategia metodológica: La Solución de Problemas como base al desarrollo psicocognitivo y al desarrollo de la personalidad. Uso de medios tecnológicos con fines pedagógicos. Plan de Estudios: Formación humanista, en ciencias, profesional y relación teórico práctica. Estrategia metodológica. La carrera favorecerá estrategias que permitan al estudiante aprender a aprender, aprender haciendo, la resolución de situaciones significativas de la realidad de la profesión. profesión, procedimientos necesarios para el ejercicio profesional y actitudes que permitan la formación del profesional desde una perspectiva integral.

Modelo de la UNED. Asume: Enfoque CognitivoConstructivista y plantea objetivos como guía, conocimientos previos, aprendizaje significativo. En el contenido curricular conciben como conceptos, hechos, generalizaciones, leyes, principios, propios de la 
Enfoques en los Modelos Educativos, Planes de Estudio y su Correspondencia con la Planeación Didáctica (Diseño Instruccional) en la Educación a Distancia,

Rosita Ulate

curriculares. Para empezar la revisión propuesta se escogió el Plan de Estudios del Programa de Protección y Manejo de los Recursos Naturales en vista de que se contaba con bastante información recopilada, producto de estudios previos de las giras de campo. Este segundo paso continuaría con la verificación de la correspondencia del modelo pedagógico y seguidamente una comparación del enfoque predominante en la carrera vs. Diseños curriculares de los cursos con giras de campo y el uso de modelos en la realización de la actividad de aprendizaje.

\section{Diseños curriculares y estrategias de aprendizaje en PROMARENA}

Como parte de las observaciones recolectadas en varias actividades de estudios realizados para otras investigaciones relacionas con el tema de actividades de aprendizaje como las giras de campoiii en un modelo de educación a distancia, se llegó a determinar una serie de deficiencias técnico pedagógicas debido a la inexistencia de diseños instruccionales para giras de campo como estrategias de aprendizaje. Según Ulate (2010):

Los diseños curriculares de los cursos son estrategias generales para los cursos, pero no profundizan en la ejecución de las estrategias y actividades de aprendizaje. El aporte de los diseños curriculares está en el planteamiento de la estructura general del diseño, ejecución y evaluación del curso, de manera que lograra una concordancia con el plan de estudios al cual pertenece. Algunos de los principales aspectos que se desarrollan en el diseño curricular de los cursos con giras de campo son: el número de créditos, código y nombre del curso, distribución de horas de estudio, bibliografía a consultar, estructura general del diseño, la ejecución y la evaluación del curso, así como el planteamiento de ejes transversales (p.4).

Otros aspectos señalados, en ese mismo estudio de las giras de campo, indica que no se encontró en ninguno de los seis cursos un desglose de diseño pedagógico o instruccional que guie y establezca los parámetros e indicadores de calidad para la realización de las giras de campo. 
Enfoques en los Modelos Educativos, Planes de Estudio y su Correspondencia con la Planeación Didáctica (Diseño Instruccional) en la Educación a Distancia,

Rosita Ulate

Es en las orientaciones del curso, información dirigida para el estudiante, se hace una descripción del propósito y algunos detalles generales de la gira de campo. No se llegó determinar la existencia de materiales de inducción, generales o específicos que se entreguen previos a la realización de la giras de campo. A la fecha, no existen lineamientos de protocolo ni estándares mínimos que guíen, dimensionen, confronten y evalúen el aprendizaje de los estudiantes en la realización de las giras.

Estudios exploratorios previos (Ulate, 2009 y 2010) indicaron claramente que no se siguen modelos de diseños instruccionales para la realización de las actividades de aprendizaje como las giras de campo. Lo más cercano son las orientaciones del curso entregadas a los estudiantes en la matrícula de cada curso. Sin embargo dichas orientaciones evidencias claramente una deficiencia en la planeación didáctica de la actividad con respecto al logro de los objetivos académicos.

Asimismo la evaluación de los aprendizajes en las giras de campo se limita a informes de gira, pruebas cortas verbales en la realización de la misma y en muy pocos casos el uso de la plataforma de aprendizaje para generar foros de realimentación con los estudiantes.

¿Qué paso con el planeamiento riguroso de las estrategias de aprendizaje y sus respectivas actividades? El gran faltante: sistemas y diseños instruccionales, pieza fundamental para una clase presencial, para la actividad fuera del aula, para la planeación de un curso en modalidad a distancia, para la producción de materiales didácticos efectivos, para el diseño de cursos que usan las plataformas de aprendizaje, total o parcialmente y para la evaluación de los aprendizajes. 
Enfoques en los Modelos Educativos, Planes de Estudio y su Correspondencia con la Planeación Didáctica (Diseño Instruccional) en la Educación a Distancia,

Rosita Ulate

\section{Evaluación de los aprendizajes en MARENA}

En la UNED la evaluación de los aprendizajes es considerado como unos procesos sistemáticos que cumple dos objetivos medulares: El primero es dar realimentación a los estudiantes sobre su progreso y verificar el aprendizaje adquirido en cuanto a conocimientos y competencias. El segundo es poder conocer los logros y determinar las dificultades que experimentan los estudiantes, con el propósito de generar medidas de acción en el mejoramiento de ese proceso sistemática de evaluación de los aprendizajes (UNED, 2009). Adicionalmente en el Plan Académico de la UNED 2001-2006 se establecen en cada Escuela unidades de Asesoría y seguimiento de la evaluación de los aprendizajes, con el propósito de "promover un estudiante creativo, crítico, investigador, humanista y comprometido con el medio ambiente y la sociedad" (UNED, 2001, pp.38-39).

En un informe de la evaluación de los aprendizajes en MARENA se establece que el proceso de evaluación de los aprendizajes en dicha carrera es "sistemático, riguroso, integrado, formativo y sumativo (...) congruente con los objetivos de aprendizaje de cada asignatura y el perfil profesional del Programa (...) ha incursionado en diseñar y aplicar estrategias alternativas para evaluar los aprendizajes de los estudiantes que evidencien un aprendizaje significativo durante todo el proceso (...) incorporando estrategias como giras, los laboratorios y sus respectivos informes, las investigaciones, proyectos de campo" (Calderón, 2009, p. 10-11). La misma autora expresa que de 134 actividades realizadas durante el 2007 al 2009 se reconoce que las más utilizadas son: a) examen con un $21.6 \%$, giras de campo con un $13,4 \%$, análisis de casos con un $14 \%$, anteproyecto de investigación con un $12 \%$ y análisis de texto y proyectos de investigación con un $10 \%$ cada una.

El reto para las mejoras realizadas en MARENA en cuanto a la evaluación de los aprendizajes, será entonces comprobar la correlación de las diferentes actividades 
Enfoques en los Modelos Educativos, Planes de Estudio y su Correspondencia con la Planeación Didáctica (Diseño Instruccional) en la Educación a Distancia,

Rosita Ulate

para cumplir con los objetivos del perfil profesional del graduado, desde el enfoque paradigmático del Programa en cuestión. De tal manera que se asegure que el conocimiento, destrezas, habilidades y actitudes hayan sido desarrolladas respetando un diseño didáctico (instruccional) de las actividades de aprendizaje bajo los parámetros del modelo pedagógico respectivo de la UNED y de la carrera. Lo que incluye también una correspondencia con los materiales didácticos utilizados, las actividades de aprendizaje y su respectiva evaluación. ¿Responde el proceso de evaluación de los aprendizajes de MARENA a la necesidad de establecer una realimentación no sólo sumativa sino también formativa ante los estudiantes?

\section{El porqué del diseño instruccional, beneficios en su uso}

Es importante en Latinoamérica establecer la definición de diseño curricular y diseño instruccional, ya que aunque complementarios, reconozco que tiende a confundirse el ámbito de acción de cada uno. El diseño curricular guía los procesos integrales, da un ordenamiento y especificaciones generales sobre un plan de estudios y sus cursos. Coll, Martín, Mauri, Miras, Onrubia, Solé y Zabala (2007) señalan claramente que una vez formulado el diseño y desarrollo curricular se enfrentan a un gran reto, en "asegurar la coherencia y la continuidad desde las formulaciones generales que suelen adoptar la primera instancia hasta su traducción en términos de propuestas concretas de actividades de enseñanza y aprendizaje en las aulas" ( $p .179)$.

Por otro lado se puede indicar que el diseño instruccional es un proceso sistemático de metodología y pedagogía que busca maximizar el aprendizaje de una materia, de una lección o de un tema por desarrollar. Al respecto la Universidad de Michigan, 2003, mencionada por Brown y Green (2011) lo definen como:

Un proceso sistemático del desarrollo de especificaciones instruccionales que aseguran su calidad a partir de las teorías de aprendizaje y de la instrucción. Proceso en el que se analiza las necesidades del estudiante, las metas y el 
Enfoques en los Modelos Educativos, Planes de Estudio y su Correspondencia con la Planeación Didáctica (Diseño Instruccional) en la Educación a Distancia,

Rosita Ulate

desarrollo que será realizado sistemáticamente al conocer las necesidades previas. Incluye el desarrollo de material instruccional y de actividades así como de la evaluación de la instrucción y de las actividades de aprendizaje (pp.5-6).

El objetivo de la implementación de un modelo o sistema de diseño instruccional radica en los principios del mismo, y son:

a) Los estudiantes son motivados a resolver problemas de la vida real,

b) activar el conocimiento previo como fundamento del nuevo conocimiento a desarrollar, c) el nuevo conocimiento es demostrado por el estudiante,

d) el nuevo conocimiento es aplicado por el estudiante y

e) el nuevo conocimiento es integrado en el mundo real del estudiante (Brown y Green, 2011).

El diseño instruccional se centra más en el estudiante que en el docente, Gustafson y Tillman (1991) describen la meta del diseño instruccional en desarrollar el conocimiento, las habilidades, destrezas y actitud del estudiante, en donde el profesor es solamente un instrumento, un vehículo para lograrlo, de tal forma que los autores indican que desde la perspectiva objetivo del diseño instruccional "the teacher may note ven need to be present during the time the learner is learning" (p.8). Ahora bien los mismos autores recomiendas hacer otra diferencia entre un modelo de diseño instruccional en el que se describe el proceso para diseñar y desarrollar la instrucción y los modelos de instrucción para el docente en el cual se especifica un formato de instrucción.

El trabajo del diseño instruccional requiere del aporte interdisciplinario de diseñadores instruccionales, expertos en evaluación, productores de materiales didácticos en cualquiera de los formatos y medios que se utilicen. De tal forma que 
Enfoques en los Modelos Educativos, Planes de Estudio y su Correspondencia con la Planeación Didáctica (Diseño Instruccional) en la Educación a Distancia,

Rosita Ulate

se requiere de una gestión y manejo del diseño instruccional en donde se distinguen fases como planeación, organización, desarrollo y control. Estas fases incluyen actividades como analizar, diseñar, desarrollo de materiales prototipos, prueba de materiales, revisión y la producción final.

A partir del análisis de los enfoques de las teorías de aprendizaje, las secuencias generales de los modelos existentes de un sistema de diseño instruccional son:

a) Análisis de la situación (necesidades, tareas y características de los estudiantes), en donde se identifican las necesidades y se establecen las metas. Uno de los aspectos más importantes en los primeros pasos del proceso de diseño instuccional es la determinación de las necesidades del profesional a formar, hay necesidades normativas, emotivas, demandadas, comparativas, anticipadas en el futuro, críticas y requeridas. Todas estas necesidades según Kaufman son determinadas dentro del sistema holístico que responde a diferentes niveles, Supra, Mega, Macro y Micro. Necesariamente pasan por un proceso de insumos, proceso, desarrollo, salida y beneficios para los diferentes niveles indicados, sean mundiales, regionales, nacionales, sectoriales, gremiales, sociales, comunales, entre otros, (Burton y Merrill, 1991). La clara definición de las necesidades en cada nivel jerárquico, posibilita el diseño curricular de un plan de estudios pertinente y asegura la identificación de diseños instruccionales que den solución a los problemas existentes en la realidad de la práctica profesional.

b) Organización de la estructura del curso o actividad, significa establecer el nivel del curso o tema, la secuencia de actividades a realizar, los métodos, materiales y medios a usar.

c) Traducción de las metas en objetivos. La teoría del diseño instruccional hace una clara diferencia entre meta y objetivo, por ejemplo Yelon (1991), describe la meta 
Enfoques en los Modelos Educativos, Planes de Estudio y su Correspondencia con la Planeación Didáctica (Diseño Instruccional) en la Educación a Distancia,

Rosita Ulate

como los lineamientos generales de lo que un estudiante deberá ser capaz de realizar en una situación del mundo real una vez finalizada la instrucción. Mientras que objetivos son lineamientos específicos de lo que un estudiante debe ser capaz de realizar dentro de una situación representativa, de acuerdo con los estándares del mundo real una vez terminada la instrucción.

d) Preparación del ambiente de aprendizaje. Según Brown y Cocking mencionados por Brown y Green (2011), hay cuatro perspectivas de desarrollo del ambiente de aprendizaje: a) centrado en el estudiante, b) centrado en el conocimiento, c) centrado en la evaluación y d) centrado en la comunidad (mezcla del centrado en el estudiante, en la evaluación y en el conocimiento).

e) Análisis de los objetivos específicos instruccionales. De acuerdo con Mager es la redacción de objetivos en términos de acción, condición y criterios (Brown y Green, 2011). Mientras que para Dick, Carey y Carey los objetivos se establecen a través de dos posibles sistemas: el primero basado en los criterios de expertos en la materia y el segundo por la determinación del desarrollo de aproximación tecnológica. En ambos sistemas se establecen metas y se realiza un análisis de habilidades subordinadas, para llegar a la determinación de los objetivos específicos de la instrucción, contemplando habilidades de entrada necesarios para iniciar la instrucción. Por otro lado Smaldino, Lowther y Russell (2008) sugieren el sistema de ABCD (Audience: descripción de los estudiantes; Behavior: la actitud esperada después de la instrucción; Conditions: las circunstancias en que el aprendizaje ocurrirá y Degree: explicación del estándar de desarrollo aceptable para el estudiante). También existen otros sistemas como el ASSURE (Análisis de los estudiantes, establecimiento de objetivos, selección de métodos instruccionales, medios y materiales, utilización de medios y materiales, requiere participación de los estudiantes y evaluación y revisión). Otros conocidos son el Diseño Instruccional de Gagnè y Brigss y el Diseño Instruccional de Davis. 
Enfoques en los Modelos Educativos, Planes de Estudio y su Correspondencia con la Planeación Didáctica (Diseño Instruccional) en la Educación a Distancia,

Rosita Ulate

f) Diseño de la estrategia de instrucción. Una de las ventajas del diseño instruccional es basarse en un modelo maestro a partir del cual enfocar cada uno de los pasos del desarrollo de un curso, una lección, un tema, etc. De no seguir un modelo se tiende a perder la secuencia y el enfoque integral de cada una de las acciones en el proceso de enseñanza y aprendizaje. Es a partir de la escogencia de este modelo maestro (al que yo llamaría como la correspondencia con el modelo educativo y enfoque de aprendizaje base del plan de estudios), que se determinan los objetivos. Los más utilizados hasta el momento han sido por la nivel en el proceso de desarrollo de habilidades de Bloom (conocer, comprender, aplicar, analizar, sintetizar y evaluar); o los que Gagné presenta como desarrollo de capacidades (habilidades intelectuales, información verbal, estrategias cognitivas, habilidades motoras y actitudes).

Gagné establece como estrategia de la instrucción algunos pasos, tales como ganar la atención del estudiante, informar los objetivos de aprendizaje, estimular las prioridades de aprendizaje, presentar un estímulo, proveer una guía al estudiante, obtener la aplicación del estudiante, promover la realimentación, evaluación del rendimiento del estudiante y mejorar la retención y transferencia del aprendizaje.

g) Diseño de los módulos o partes de la lección sea para una lección presencial, instrucción programada o para un modelo de educación a distancia. Al respecto se distinguen dos categorías del diseño de la instrucción: la primera con realimentación inmediata (entre profesor y estudiante) o con inmediata realimentación para el estudiante que estudia solo.

Se identifican las posibles actividades instruccionales, aspecto práctico de interés para los docentes y productores de materiales didácticos. Puede ser basado en la resolución de problemas, en la simulación, en juegos instruccionales, enseñanza justo a tiempo. Asimismo se distinguen prácticas instruccionales explicadas por 
Enfoques en los Modelos Educativos, Planes de Estudio y su Correspondencia con la Planeación Didáctica (Diseño Instruccional) en la Educación a Distancia,

Rosita Ulate

Joyce y Weil, así como Ellis y Fouts mencionados por Brown y Green (2011), entre ellos, actividades basadas en la investigación, en la comparación, en la evaluación, así como en aspectos personales del estudiante, en su proceso de información, conducta y interacción social.

h) Conducir una evaluación formativa. En cuanto los términos de actitud observables en el estudiante, el diseño instruccional también contempla varios, como por ejemplo, términos de observación, términos de ejecución, términos de operación y términos de evaluación.

i) Implementación del tema, curso, lección a desarrollar. Brown y Green (2011) también recomiendan actividades instruccionales como identificación de diferencias y similitudes, clasificación, resumen, tomar notas, reforzamiento del esfuerzo y proveer reconocimientos, premios y símbolos de reconocimiento, tareas y prácticas, representaciones lingüísticas, aprendizaje colaborativo, establecimiento de objetivos y dar realimentación, generalización y prueba de hipótesis, preguntas, misterios y organización avanzada.

j) Evaluación final generalmente sumativa. Brown y Green (2011) recomiendan tener en cuenta dos aspectos validez y viabilidad. La validez toma en cuenta como es presentada la evaluación a los estudiantes, si es razonable, bien explicada, si se presenta datos apropiados. Mientras que la viabilidad si se toma en cuenta el campo de dominio estudiado en las evaluaciones.

La evaluación sumativa deberá responder a los objetivos instruccionales planteados previamente. Es por eso que objetivos instruccinales bien planteados darán como resultado conocer si el estudiante demuestra el conocimiento, la habilidad, actitud o destreza esperada al completar la instrucción. 
Enfoques en los Modelos Educativos, Planes de Estudio y su Correspondencia con la Planeación Didáctica (Diseño Instruccional) en la Educación a Distancia,

Rosita Ulate

En cuanto al desarrollo de la evaluación sumativa para promover un cambio en el conocimiento Millman y Greene, así como Hopkins mencionados por Brown y Green (2011) establecen una guía de preguntas para la creación de un plan de evaluación acertada en las pruebas o evaluaciones a estudiantes. Presentan la posibilidad de usar en las pruebas: falso y verdadero, elección múltiple, asociación de ítems, respuesta de ítem corta, construcción de respuestas en el test, ítems fáciles. Mientras para un cambio en las habilidades, Brown y Green (2011) recomiendan el uso de preguntas directas, rango de desarrollo de actividades, observación, sistematización de anécdotas, grabaciones, portafolios, rúbricas. Por otro lado los mismos autores en cuanto a un cambio en la actitud recomiendan, encuestas, cuestionarios, bitácora de un reporte personal, entrevistas, entre otras.

Por último en cuanto a la evaluación sumativa, se distinguen tres periodos comparativos, esto es antes, durante y después de la instrucción, aspectos que enriquecen el poder conocer el rendimiento del estudiante y la efectividad del diseño de la instrucción. De tal forma que un sistema de diseño instruccional responsable tendría implementado un reporte final de un plan instruccional, que debería contemplar aspectos como un resumen ejecutivo, el propósito de la evaluación, la metodología usada, los resultados obtenidos, conclusiones y recomendaciones.

Los pasos generales de un sistema de diseño instruccional varían de acuerdo al modelo a utilizar. Luzargo (2004) hace una detallada exposición y explicación a partir de una clasificación por generaciones de desarrollo de los diseños instruccionales (véase Tabla 2).

Tabla 2: Resumen de las características de los diseños instrtuccionales por generación. Adaptado de Luzargo (2004, pp. 13-16)

\begin{tabular}{c|l|l}
\hline Generación & \multicolumn{1}{|c|}{ Modelo } & \multicolumn{1}{c}{ Características. } \\
\hline Primera (1960) & Glaser (1966) & $\begin{array}{l}\text { Están basados en el enfoque conductista. Se formula linealmente el } \\
\text { desarrollo de la instrucción. Son sistemáticos. Prescriben los métodos } \\
\end{array}$ \\
& & $\begin{array}{l}\text { específicos y programados, centrados en el conocimiento y destrezas de } \\
\text { tipo académico, y en la formulación de objetivos de aprendizajes }\end{array}$ \\
\hline
\end{tabular}


Enfoques en los Modelos Educativos, Planes de Estudio y su Correspondencia con la Planeación Didáctica (Diseño Instruccional) en la Educación a Distancia,

Rosita Ulate

\begin{tabular}{|c|c|c|}
\hline & & $\begin{array}{l}\text { observables y secuenciales. Los principios fundamentales son la } \\
\text { descomposición de las informaciones en unidades muy pequeñas. Las } \\
\text { actividades se basan en dar respuestas y el uso de refuerzos, según los } \\
\text { medios utilizados. }\end{array}$ \\
\hline $\begin{array}{l}\text { Segunda } \\
\text { (1970) }\end{array}$ & $\begin{array}{l}\text { IDI (1971) } \\
\text { Kaufman (1972) } \\
\text { Gagne y Briggs (1974) } \\
\text { IPISD (1975) } \\
\text { Crittendon y Massey (1978) } \\
\text { Roberts (1978) } \\
\text { Briggs y Wagner (1979) }\end{array}$ & $\begin{array}{l}\text { Tienen su fundamento en los } \mathrm{DI} \text { de la primera generación pero } \\
\text { desarrollados como macro-procesos. Son sistemas más abiertos, en } \\
\text { donde se toman en cuenta aspectos internos y externos de la instrucción } \\
\text { Permitan una mayor participación cognitiva por parte del estudiante. Se } \\
\text { fundamentan en la teoría de sistema y la del procesamiento de la } \\
\text { información. Son diseños instruccionales de transición. Poseen mayor } \\
\text { interactividad. Están centrados tanto en la enseñanza como en el } \\
\text { aprendiz. Se ubican más en el proceso que en el producto. En la medida } \\
\text { en que se van desarrollando, se integran las fases entre sí. Guardan la } \\
\text { característica lineal entre sus fases independientes. }\end{array}$ \\
\hline Tercera (1980) & $\begin{array}{l}\text { Romizowski (1981) } \\
\text { Kemp (1985) } \\
\text { Diamond (1989) } \\
\text { Dick y Reiser (1989) } \\
\text { Gerlach y Ely (1989) } \\
\text { Van Patten (1989) }\end{array}$ & $\begin{array}{l}\text { Han sido llamados también DI cognitivos. Desarrollan prescripciones } \\
\text { explícitas de las acciones instruccionales, que enfaticen la comprensión } \\
\text { de los procesos de aprendizaje. Forman parte de los diseños planteados } \\
\text { por Merrill (1994). Las estrategias son heurísticas. Los contenidos pueden } \\
\text { ser planteados como tácitos. Los conocimientos deben ser de tipo } \\
\text { conceptual, factual y procedimental, basados en la práctica y resolución } \\
\text { de problemas. Interactividad más orientada al uso y a la aplicación de } \\
\text { simulaciones. Énfasis en el estudio de los niveles mentales de los } \\
\text { alumnos y de la estructura cognitiva. Toman en consideración el modelo } \\
\text { mental, para hacer corresponder la transacción instruccional, así como el } \\
\text { dominio del conocimiento. El uso de tecnologías como el computador } \\
\text { maximiza el aprendizaje y abre oportunidades de diálogo para el } \\
\text { estudiante. Los objetivos instruccionales son más integrales. El } \\
\text { aprendizaje cooperativo, la indagación y el aprender a aprender son } \\
\text { altamente mediados por las tecnologías de la información y la } \\
\text { comunicación. }\end{array}$ \\
\hline Cuarta (1990) & $\begin{array}{l}\text { Seals y Glasgow (1990) } \\
\text { Dick y Carey (1990) } \\
\text { Berman y Moore (1990) } \\
\text { Rapid Prototyping (1990) } \\
\text { Chaos (1991) } \\
\text { Layer of Necessity (1991) } \\
\text { Leshin, Pollack, Reigieluth (1992) }\end{array}$ & $\begin{array}{l}\text { No prescriben el aprendizaje a lograr, por cuanto el conocimiento no es } \\
\text { único. Están fundamentados sobre la primicia de que existen diversos } \\
\text { mundos epistemológicos. Se caracteriza por sustentarse en las teorías } \\
\text { constructivista, la del caos, la de los sistemas, lo cual da como resultado } \\
\text { un modelo heurístico. Tienden a que el diseñador descubra la } \\
\text { combinación de materiales y actividades de enseñanza que orienten al } \\
\text { alumno a darse cuenta del valor del descubrimiento para futuros } \\
\text { aprendizajes. El diseño instruccional, desde esta perspectiva, privilegia la } \\
\text { habilidad del alumno para crear interpretaciones por sí mismo y manipular } \\
\text { las situaciones hasta que las asuma como proceso de aprendizaje. Están } \\
\text { centrados en el proceso de aprendizaje y no en los contenidos } \\
\text { específicos. }\end{array}$ \\
\hline Quinta (1999)iv & $\begin{array}{l}\text { Analyze, Design, Develop, } \\
\text { Implement and Evaluate (ADDIE), } \\
\text { (Maribe, 2009), } \\
\text { Modelo constructivista, Modelo } \\
\text { educativa ACAD (Acedo, 2003). } \\
\text { Hannafin y Peck, } \\
4 \text { MAT (Mc Carthy \& Mc Carthy, } \\
\text { 2006). }\end{array}$ & $\begin{array}{l}\text { "El diseño instruccional se concibe como el núcleo del proceso educativo } \\
\text { en la educación virtual y en sí mismo como un proceso sistemático, } \\
\text { planificado y estructurado donde se produce una variedad de materiales } \\
\text { educativos adaptados a las necesidades de los educandos, asegurándose } \\
\text { así la calidad del aprendizaje" (Luzardo, 2004, p.44). }\end{array}$ \\
\hline
\end{tabular}


Enfoques en los Modelos Educativos, Planes de Estudio y su Correspondencia con la Planeación Didáctica (Diseño Instruccional) en la Educación a Distancia,

Rosita Ulate

ASSURE (Smaldino, Lowther,

Russell, 2008).

Fuente: Elaboración propia 2011. Adaptado de Luzargo (2004, pp. 13-16) y Polo (2001, p.p. 1-10)

De acuerdo con el enfoque ecléctico postmoderno, los sistemas de diseño instruccional deben adaptarse a: a) el uso alternativo de medios de comunicación y tecnológicos disponibles para el estudiante; b) la particularidades de los estilos de aprendizaje de cada estudiante; c) la incorporación de los paradigmas cognitivo y constructivista que no necesariamente siguen un modelo lineal de instrucción y d) las diferentes interpretaciones y perspectivas de las experiencias individuales en un ambiente determinado (Brown y Greene, 2011).

\section{Lo que interesa a los educadores}

Los docentes que apoyan los procesos de enseñanza y aprendizaje no necesariamente participan en cada uno de las fases de diseño del plan de estudios, del diseño curricular o instruccional y no necesariamente participan colegiadamente en el diseño de la evaluación de los aprendizajes. Entonces mi gran preocupación, lejos de sistemas lineales y no flexibles, es qué pasa si los profesores no cuentan con las respectivas indicaciones mínimas, lineamientos o estándares mínimos en el desarrollo de sus actividades docentes. El diseño instruccional no es sólo un plan académico a llevar a cabo para los estudiantes, es también para los docentes. Los docentes y los estudiantes requieren conocer: ¿Cuál es el propósito del plan de estudios, del curso, de la actividad de aprendizaje, de la evaluación a realizar? ¿Qué se espera de ellos?

\section{Conclusiones}

Al estudiar los enfoques paradigmáticos de cada uno de los programas de estudio de la ECEN, específicamente el de PROMARENA vs. las actividades de aprendizaje 
Enfoques en los Modelos Educativos, Planes de Estudio y su Correspondencia con la Planeación Didáctica (Diseño Instruccional) en la Educación a Distancia,

Rosita Ulate

como las giras de campo se llega a la conclusión de que no hay una correspondencia entre las partes.

Asimismo se demuestra que las estrategias de aprendizaje no están claramente diseñadas a través de modelos de instrucción que respondan a los enfoques paradigmáticos y corrientes de las diferentes teorías de aprendizaje que dice seguir bajo un modelo ecléctico.

El avance en la investigación exploratoria y empírica realizada específicamente en PROMARENA, marca un gran reto en la el proceso de enseñanza y aprendizaje a distancia. Aunque no se ha terminado, temo conocer el resultado final, hemos avanzado en el modelo pedagógico y su correspondencia con los planes de estudios hasta el diseño curricular de los cursos, sin embargo la correlación de las estrategias, actividades y evaluación de aprendizaje ha recaído en una desorientación ecléctica que no nos permite ser efectivo con la entrega de profesionales acorde con las necesidades de la sociedad.

\section{Recomendaciones}

Los profesionales que soportan la oferta de un plan de estudios universitario, desde los directores, encargados de programa, de cátedra, profesores, diseñadores curriculares, productores de materiales, diseñadores instruccionales y demás dependencias de apoyo académico deben tener claridad en los paradigmas pedagógicos que conforman la carrera.

Se recomienda el establecimiento de objetivos en los diseños instruccionales de las actividades de aprendizaje de PROMARENA, para así generar una correspondencia en las metas, objetivos, desarrollo, producción y evaluación de la gestión académica a realizar. 
Enfoques en los Modelos Educativos, Planes de Estudio y su Correspondencia con la Planeación Didáctica (Diseño Instruccional) en la Educación a Distancia,

Rosita Ulate

Aprovechar el uso de las tecnologías y recursos de la información y la comunicación con que cuenta la UNED, para diseñar modelos que respondan a las necesidades de la sociedad en la formación de futuros profesionales y que se conviertan en verdaderos centros de aprendizaje y no solamente repositorios de contenidos, tareas, foros y trabajos de investigación sin ningún nexo y conexión con las competencias, habilidades, destrezas, conocimientos y actitudes a desarrollar en los estudiantes.

Se aconseja generar un estudio del plan de estudios por etapas de desarrollo y determinar que enfoques de las teorías del aprendizaje son oportunos en cada nivel o curso para así, poder encontrar modelos de instrucción que se ajusten a los estilos de aprendizaje y al desarrollo del mismo en los estudiantes.

Los profesionales que soportan la oferta de un plan de estudios universitario, desde los directores, encargados de programa, de cátedra, profesores, diseñadores curriculares, productores de materiales, diseñadores instruccionales y demás dependencias de apoyo académico deben tener claridad en los paradigmas pedagógicos que conforman la carrera.

Debe hacer una clara correspondencia entre los paradigmas dominantes de los planes de estudio, los enfoques a utilizar desde las teorías de aprendizaje y que estos se reflejen en el diseño curricular, el diseño instruccional y la evaluación de los aprendizajes.

Es necesario revisar y estudiar los diferentes modelos de los sistemas instruccionales existentes y rescatarlos desde sus diferentes corrientes teóricas, sea conductismo, cognitivismo, constructivismo, entre otros. 
Enfoques en los Modelos Educativos, Planes de Estudio y su Correspondencia con la Planeación Didáctica (Diseño Instruccional) en la Educación a Distancia,

Rosita Ulate

Con el uso de las tecnologías de la información y la comunicación como vehículos para la entrega de la docencia, se deben utilizar métodos instruccionales que aseguren la calidad del uso de actividades y estrategias a través de las plataformas de aprendizaje.

No puedo más que solicitar volver a rescatar los sistemas de diseño instruccional, dentro de modelos para cualquiera de los paradigmas y enfoques de la teoría del aprendizaje, sea conductista, cognitivista, constructivista o una mezcla de ellos, ordenada y responsablemente. Más aún cuando las innovaciones de la comunicación y la tecnología nos marca nuevos derroteros para llegar por nuevos medios y formas al estudiante. Me opongo a pensar en que de no recuperar las buenas prácticas de los sistemas de diseño instruccional y sus modelos, seamos responsables de una alta entropía en los procesos de enseñanza y aprendizaje. Como se diría en economía ecológica, seríamos responsables de basureros de información y materiales que no tienen ningún sentido. Por algo al principio del Génesis dice: en el principio nada tenía forma, todo era un mar profundo de oscuridad... entonces Dios creo el cielo y la tierra... y hubo luz y era buena.

\section{Bibliografía}

Azze, A. (2011). Análisis de las particularidades del Modelo Pedagógico por Carreras de la ECEN. [Sin publicar]. San José, Costa Rica: Comisión Seguimiento al Modelo Pedagógico en la ECEN. Escuela de Ciencias Exactas y Naturales.

Brown, A \& Green, T. (2011). The essentials of instruccional design. Connecting fundamental principles with process and practice. Boston. MA: Pearson Education. Inc.

Burton, J. \& Merrill, P. (1991). Chapter 2. Needs assessment: Goals, needs and Priorities. (15-43). En Briggs, L., Gustafson, K. \& Tillman, M. Intructional Design: Principies and applications. (2a. Ed.)Englewood Cliffs, New Jersey, EE.UU: Educational Technology Publications. 
Enfoques en los Modelos Educativos, Planes de Estudio y su Correspondencia con la Planeación Didáctica (Diseño Instruccional) en la Educación a Distancia,

Rosita Ulate

Calderón, Y. (2009). Informe acerca de la evaluación de los aprendizajes en el Programa de Protección y Manejo de Recursos Naturales (MARENA). San José, Costa Rica: ECEN.

Coll, C., Martín, E., Mauri, T., Miras, M., Onrubia, J., Solé, I y Zabala, A. (2007). El constructivismo en el aula. (18 ed.). España: Editorial GRAO.

Cursi, R. (s.f.). Futuras acciones para incorporar las competencias y la práctica de las mismas en la educación a distancia. Caso Universidad Metropolitana. Recuperado el 4 de mayo 2011 de http://www.unica.edu.ve/fpd/memorias/30012009/5/Renata\%20Curci\%20\%20Ponencia.pdf

Gustafson, K. \& Tillman, M. (1991). Chapter 1. Introduction. (3-16). En Briggs, L., Gustafson, K. \& Tillma, M. Intructional Design: Principies and applications. (2‥ Ed.)Englewood Cliffs, New Jersey, EE.UU: Educational Technology Publications.

Luzargo, H.J. (2004). Informe No. 3. Herramientas nuevas para los ajustes virtuales de la educación. Análisis de modelos de diseño instruccional para eventos educativos en línea. Accelerated Degree Program Doctorate of Education in Technology Education. Tecana American University. Mérida, México: Recuperado el 4 de mayo 2011 de http://www.tauniversity.org/tesis/Tesis_Hendry_Luzardo.pdf

Maribe, R. (2009). Instruccional design. The ADDIE approach. Athens, GA, EE.UU: Springer Science and Business Media.

Mc Charthy, B. \& Mc. Carthy, D. (2006). Teaching around the 4MAT Cycle. Designing Instruction for diverse learners with diverse learning styles. CA., EE.UU: Crowin Press. A Sage Publications Co.

Merill, D. (2008). This is a brief introduction to Dr. Merrill's thoughts about instructional design. (Video). Recuperado el 29 de mayo del 2011 de http://www.youtube.com/watch?v=i TKaO2-jXA

Polo, M. (2001). El diseño instruccional y las tecnologías de la información y la comunicación. Docencia Universitaria. II (2). Venezuela. SADPRO-UCV.

Smaldino, S., Lowther, D. \& Russell, J. (2008). Instruccional technology and media for learning. (9a. Ed.) .EE.UU: Pearson Merrill Prentice Hall. 
Enfoques en los Modelos Educativos, Planes de Estudio y su Correspondencia con la Planeación Didáctica (Diseño Instruccional) en la Educación a Distancia,

Rosita Ulate

Ulate, R. (2009). Valoración Participativa de las Características Técnico Pedagógicas y Usos Académicos de un Material Multimedial para las Giras de Campo. [Sin publicar]. San José, Costa Rica: NOVA.

Ulate, R. (2010). Las giras de campo en PROMARENA. [Informe]. San José, Costa Rica: Escuela de Ciencias Exactas y Naturales.

UNED. (2001). Plan Académico UNED 2001-2006. San José, Costa Rica: UNED.

UNED (2005). Reglamento de la Gestión Académica de la UNED. San José, Costa Rica: UNED.

Yelon, S. (1991). Chapter 4. Writing and using intruccional objectives. (75-122). En Briggs, L., Gustafson, K. \& Tillma, M. Intructional Design: Principies and applications. (2 ${ }^{\mathrm{a}}$. Ed.)Englewood Cliffs, New Jersey, EE.UU: Educational Technology Publications.

\footnotetext{
i La comisión integrada para estudio de varias áreas de la docencia: Aida Azze (programas de estudio), Rosita Ulate (diseños curriculares y diseño instruccional) y Yency Calderón (evaluación de los aprendizajes).

ii La formación humanista incluye estudios generales y ejes transversales.

iii López, Arévalo, Rodríguez, Andrade-Piedra y Quirós (2008) reconocen en las giras de campo la combinación de elementos teórico-prácticos y la participación de los actores directamente involucrados en las experiencias o visitas. Ellos indican que en éstas se genera el intercambio de conocimientos, el análisis, la reflexión y la evaluación.

iv La quinta generación es expuesta por la autora.
} 